\title{
Antioxidant Effect of Annexin A-1 Induced by Low-dose Ionizing Radiation in Adipose-derived Stem Cells
}

\author{
Ji-Eun You ${ }^{1, *}$, Seung-Wan Lee ${ }^{2, * *}$, Keun-Sik Kim ${ }^{1, * *}$ and Pyung-Hwan Kim ${ }^{1, ;, * *}$ \\ ${ }^{1}$ Department of Biomedical Laboratory Science, Konyang University, Daejeon 35365, Korea \\ ${ }^{2}$ Department of Radiological Science, Konyang University, Daejeon 35365, Korea
}

\begin{abstract}
Radiation therapy is one of the primary options for the treatment of malignant tumors. Even though it is an effective anti-cancer treatment, it can cause serious complications owing to radiation-induced damage to the normal tissue around the tumor. It was recently reported that normal stem cell response to the genotoxic stress of ionizing radiation can boost the therapeutic effectiveness of radiation by repairing damaged cells. Therefore, we focused on annexin A-1 (ANXA1), one of the genes induced by low-dose irradiation, and assessed whether it can protect adipose-derived stem cells (ADSCs) against oxidative stress-induced damage caused by low-dose irradiation and improve effectively cell survival. After confirming ANXA1 expression in ADSCs transfected with an ANXA1 expression vector, exposure to hydrogen peroxide $\left(\mathrm{H}_{2} \mathrm{O}_{2}\right)$ was used to mimic cellular damage induced by a chronic oxidative environment to assess cell survival under oxidative conditions. ANXA1-transfected ADSCs demonstrated that increased viability compared with un-transfected cells and exhibited enhanced anti-oxidative properties. Taken together, these results suggest that ANXA1 could be used as a potential therapeutic target to improve the survival of stem cells after low-dose radiation treatment.
\end{abstract}

Key Words: ADSC, ANXA1, Low-dose radiation, Oxidative stress

\section{INTRODUCTION}

Cancer is a disease caused by genetic mutations and is among the leading causes of death worldwide. Surgery, chemotherapy, and radiation therapy are the most common methods used to treat cancer. Surgery is the most preferred for solid cancers, and chemotherapy and radiation therapy are used to treat advanced-stage cancers or to prevent recurrence after surgery. These treatments can be used alone or in combination to treat lesions.

Radiation therapy utilizes high-energy electromagnetic radiation to retard the growth of or kill cancer cells, as rapidly proliferating cancer cells are more sensitive to DNA damage than normal cells (Hur and Yoon, 2017; Liauw et al., 2013). However, during radiation therapy, normal cells neighboring the tumor also receive a considerable dose of ionizing radiation. This exposure can cause damage to the healthy tissues, which may manifest immediately or later, if the patient survives. In addition, radiation therapy also has side effects such as hormonal imbalance and diminished bone marrow function, via damaging healthy cells (Baskar et al., 2012). Low linear energy transfer (LET) radiation (X-rays, -rays, and particles) generate free radicals and reactive oxygen

Received: October 15, 2020 / Revised: December 7, 2020 / Accepted: December 9, 2020

* Graduate student, ${ }^{* *}$ Professor.

${ }^{\dagger}$ Corresponding author: Pyung-Hwan Kim. Department of Biomedical Laboratory Science, Konyang University, 158 Gwanjeodong-Ro, Seo-Gu, Daejeon 35365 , Korea

Tel: +82-42-600-8436, Fax: +82-42-600-8408, e-mail: kimph1010@konyang.ac.kr

(C) The Korean Society for Biomedical Laboratory Sciences. All rights reserved.

(c) This is an Open Access article distributed under the terms of the Creative Commons Attribution Non-Commercial License (http://creativecommons.org/licenses/by-nc/3.0/) which permits unrestricted non-commercial use, distribution, and reproduction in any medium, provided the original work is properly cited. 
(A)

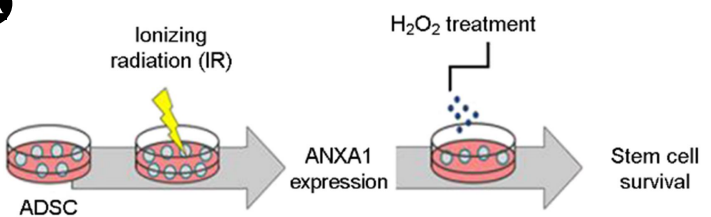

B

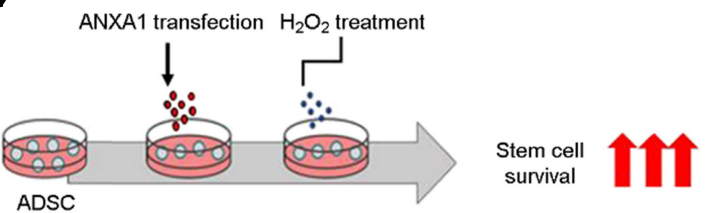

Fig. 1. Schematic diagram of the proposed function of ANXA1 expressing in ADSCs against oxidative stress. (A) ANXA1 function induced by low-dose ionizing radiation and (B) Increased cell survival of ANXA1 overexpressing ADSC under oxidative stimulation conditions.

species (ROS) such as superoxide $\left(\mathrm{O}_{2}\right)$ and hydroxyl radical $(\mathrm{OH})$ as well as non-radical molecules such as hydrogen peroxide $\left(\mathrm{H}_{2} \mathrm{O}_{2}\right)$ and singlet oxygen $\left(\mathrm{O}_{2}\right)$. Intracellular ROS can cause oxidation of biological macromolecules and activation of intracellular signaling pathways, which induce apoptosis and cell cycle arrest (Hur and Yoon, 2017; Seifried et al., 2007; Buldak et al., 2015). ROS induced by low LET radiation is strongly associated with oxidative stress, which is detrimental to human health. Overproduction of $\mathrm{H}_{2} \mathrm{O}_{2}$, the most common ROS, may lead to oxidative stress, which may in turn result in permanent changes in cells, leading to loss of function of proteins and disease (Onmaz et al., 2011). Thus, intracellular redox potential can influence cellular functions, and its dysregulation is associated with disease (Bentzen, 2006; Beerman, 2017).

Although radiation therapy prolongs patient survival, the damage it causes to normal tissue remains an important clinical concern. In particular, resident stem cells around the lesion can also be damaged. However, recently, it has been reports that low-dose radiation enhances the functioning of stem cells (Mohrin et al., 2010).

Therefore, we assessed how low-dose radiation affects stem cell survival and focused on ANXA1, one of the genes activated by radiation.

ANXA1 is a ubiquitous $37-\mathrm{kDa}$ protein that belongs to the family of calcium/phospholipid-binding proteins (Lim and Pervaiz, 2007). It is expressed in various tissues and is involved in several biological processes such as calcium signaling, membrane aggregation, inflammation, phagocytosis, cell proliferation, apoptosis, and tumor progression (Beerman, 2017). In this study, we evaluated how ANXA1 affects the biological function of adipose-derived stem cells (ADSCs) exposed to low-dose radiation (Fig. 1).

\section{MATERIALS AND METHODS}

\section{Cell culture}

ADSCs were kindly provided by Dr. Jae-Yeol Cho at Seoul National University in Seoul, Republic of Korea. The ADSCs were cultured in Dulbecco's modified Eagle's medium with high glucose (Hyclone, Logan, UT) containing $10 \%$ fetal bovine serum (Hyclone) at $37^{\circ} \mathrm{C}$ in a $5 \% \mathrm{CO}_{2}$ incubator.

\section{X-ray irradiation}

ADSCs were seeded in a $35 \mathrm{~mm}$ dishes $\left(9 \times 10^{4}\right.$ cells $)$ and then cultured for $24 \mathrm{~h}$ and irradiated. An X-ray radiographic system (APOLLON, GEMSS, Republic of Korea) was used to irradiate the ADSC cells. The ADSC cells were directly exposed to X-rays at a tube voltage of $120 \mathrm{kVp}$ with a tube current of $320 \mathrm{~mA}$. The radiation dose from a single X-ray exposure was $0.02 \mathrm{mGy}$, and a total radiation dose of $1 \mathrm{mGy}$ was delivered to the ADSC cells.

RNA extraction and the reverse transcription-PCR (RT$\mathrm{PCR}$ ) assay and conventional polymerase chain reaction (PCR)

ADSCs were seeded in 6-well plates $\left(9 \times 10^{4}\right.$ cells/well $)$ and harvested after irradiation ( $1 \mathrm{mGy}$ ) or $\mathrm{H}_{2} \mathrm{O}_{2}$ (hydrogen peroxide) treatment at $60 \mathrm{mM}$ for $4 \mathrm{~h}$. Total RNA was isolated from the cells using Trizol reagent (Invitrogen, California, USA) following the manufacturer's manual. Complementary DNA (cDNA) was synthesized using the DiaStar $2 \times$ RT Pre-Mix (Solgent, Daejeon, Republic of Korea). The synthesized cDNA was subjected to conventional PCR using a Solg $2 \times$ Taq PCR Pre-Mix (Solgent) according to the manufacturer's protocols. The PCR products were electrophoresed on 2\% agarose gels (Agarose, Molecular Biology 
Table 1. List and sequences of primers used

\begin{tabular}{cll}
\hline \hline Genes & \multicolumn{1}{c}{ Forward } & \multicolumn{1}{c}{ Reverse } \\
\hline GAPDH & AGG GCT TTT AAC TCT GGT & CCC CAC TTG ATT TTG GAG GGA \\
ANXA1 & AAT CCA TCC TCG GAT GTC GC & ACA CGT TTA CGT CTG TCC CC \\
GPX4 & GCC TTC CCG TGT AAC CAG T & GCG AAC TCT TTG ATC TCT TCG T \\
\hline
\end{tabular}

Grade, Vivantis) prepared using $1 \times$ TAE buffer (Biosesang). Gel images were obtained using a chemiluminescence analyzer (VilberLourmat, Eberhardzell, Germany). Table 1 shows the primer sequences used in this study.

\section{Generation of ANXA1-expressing vector and its trans- fection into ADSCs}

The ANXA1-expressing vector was cloned to be expressed by the cytomegalovirus promoter. Plasmid construction was confirmed by restriction digestion. The plasmid was then transformed into Escherichia coli $\mathrm{DH} 5 \alpha$ cells and purified using the Axygen ${ }^{\circledR}$ AxyPrep Plasmid Mini-prep Kit (Axygen, New York, USA). The purity and concentration of the plasmid was evaluated based on the ratio of absorbance at $260 \mathrm{~nm}$ and $280 \mathrm{~nm}$ and absorbance at $260 \mathrm{~nm}$ wavelength, respectively. ADSCs were seeded in 6 -well plates $(4 \times$ $10^{4}$ cells/well) one day before transfection. pcDNA3.1-GFP plasmid containing the ANXA1 expression cassette (Cosmogentech, Seoul, Republic of Korea) was then transfected into the cells using DOTAP (1,2-dioleoyl-3-trimethylammoniumpropane), a liposome-based delivery carrier prepared according to a previous protocol established by our laboratory (Koh et al., 2019).

\section{Cell viability assay under oxidative condition}

To assess the anti-oxidant effects of ANXA1, $\mathrm{H}_{2} \mathrm{O}_{2}$ was used to mimic the oxidative stress induced by low-dose radiation. Untreated ADSCs were used as control. First, to determine the optimal concentration of $\mathrm{H}_{2} \mathrm{O}_{2}$ to treat ADSCs with, the cells were treated with various concentrations of $\mathrm{H}_{2} \mathrm{O}_{2}$. 3-(4,5-Dimethylthiazol-2-yl)-2,5-diphenyltetrazolium bromide (MTT) $(2.5 \mathrm{mg} / \mathrm{mL}$; Sigma, UK) was used to determine cell viability after treatment with $\mathrm{H}_{2} \mathrm{O}_{2}$. Briefly, the medium in each well was removed and replaced with $300 \mu \mathrm{L}$ MTT reagent and the plates incubated at $37^{\circ} \mathrm{C}$ for
$4 \mathrm{~h}$. Following this, the supernatant was removed, and the cells were treated with dimethyl sulfoxide (Sigma-Aldrich, USA) for 15 mins at $37^{\circ} \mathrm{C}$. Absorbance was measured at $570 \mathrm{~nm}$ wavelength on VerxaMax (Associates of Cape Cod Incorporated, MA). Cell viability was normalized as a percentage using the absorbance value for the control.

\section{Statistical analysis}

Data were analyzed using paired $t$-test and one-way analysis of variance. $P<0.01$ was considered statistically significant and is indicated in the figures as $* * *$. Statistical analyses were performed using the Prism software for Windows (ver. 5.01; GraphPad Software, USA).

\section{RESULTS}

\section{ANXA1 expression induced at the transcriptomic level by low-dose radiation}

To assess whether ANXA1 expression is induced in ADSCs when irradiated with low-dose radiation, cells were seeded in a $35 \mathrm{~mm}$ dish at $3 \times 10^{5}$ cells $/ \mathrm{mL}$. $24 \mathrm{~h}$ after seeding, ADSCs were exposed to low-dose (1 mGy) radiation. The expression of ANXA1 at the transcriptome level was then assessed using cDNA synthesis analysis at $6 \mathrm{~h}$ (sample A) and $48 \mathrm{~h}$ (sample B) after irradiation. Our data indicated that ANXA1 expression was higher at the $6 \mathrm{~h}$ time point compared with that in the control group $(0 \mathrm{~h})$. Notably, ANXA1 expression returned to normal levels $48 \mathrm{~h}$ post-irradiation (Fig. 2A). These patterns were similarly observed in quantitative data (Fig. 2B). These results indicate that low-dose radiation induces transiently the expression of ANXA1 in ADSCs. 
(A)

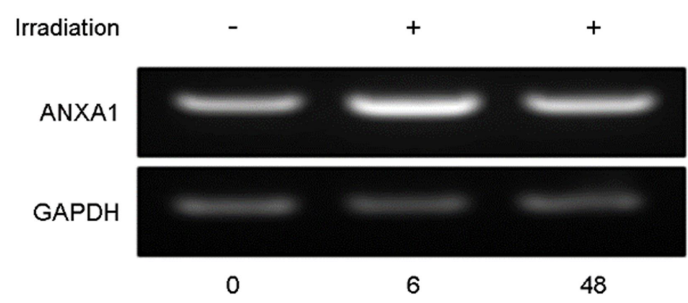

B

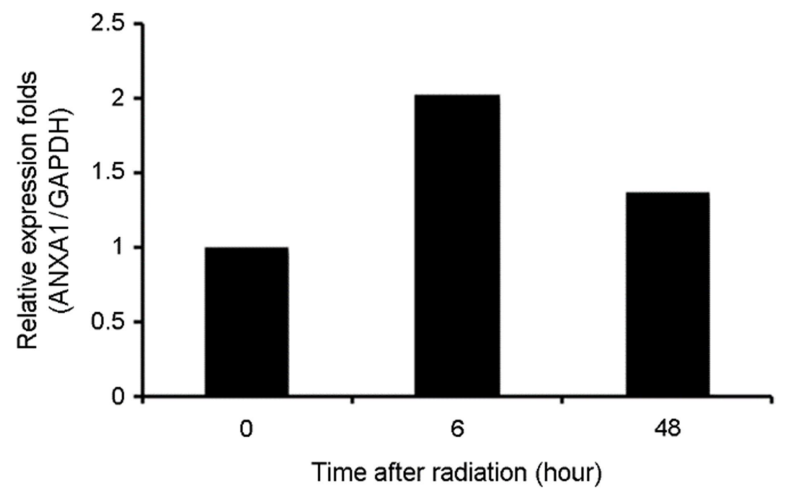

Fig. 2. The confirmation of ANXA1 expression induced by lowdose ionizing radiation. (A) ADSCs were irradiated with low-dose radiation $(1 \mathrm{mGy})$ and then the expression of ANXA1 assessed in transcriptome level at 6 and $48 \mathrm{~h}$ post-irradiation. (B) Quantified values of ANXA1 expression were normalized by GAPDH.

\section{Viability of ADSCs expressing radiation-induced ANXA1 under oxidative conditions}

To assess whether ANXA1 induced by low-dose radiation is resistant to oxidative cellular damage, cells were treated with various concentrations of $\mathrm{H}_{2} \mathrm{O}_{2}$ (from 20 to $100 \mathrm{mM}$ ) to mimic the in vivo oxidative environment. As shown in Fig. 3A, ADSC viability was inversely proportional to $\mathrm{H}_{2} \mathrm{O}_{2}$ concentration. The half maximal inhibitory concentration $\left(\mathrm{IC}_{50}\right)$ of $\mathrm{H}_{2} \mathrm{O}_{2}$ was $60 \mathrm{mM}$. To evaluate the antioxidant effects of ANXA1, ADSCs were exposed to low-dose radiation, and then cell viability was determined using MTT assay. Fig. 3B depicts the viability of ADSCs treated with low-dose radiation and $\mathrm{H}_{2} \mathrm{O}_{2}$. Our data indicated that cells treated with $60 \mathrm{mM} \mathrm{H} \mathrm{O}_{2}$ exhibited lower viability compared with controls, consistent with the result in Fig. 3A. Notably, irradiated ADSCs were observed to significantly higher viability compared with those treated with $\mathrm{H}_{2} \mathrm{O}_{2}$. However, ADSCs treated with $\mathrm{H}_{2} \mathrm{O}_{2}$ after irradiation exhibited a higher survival rate compared with cells of the
A

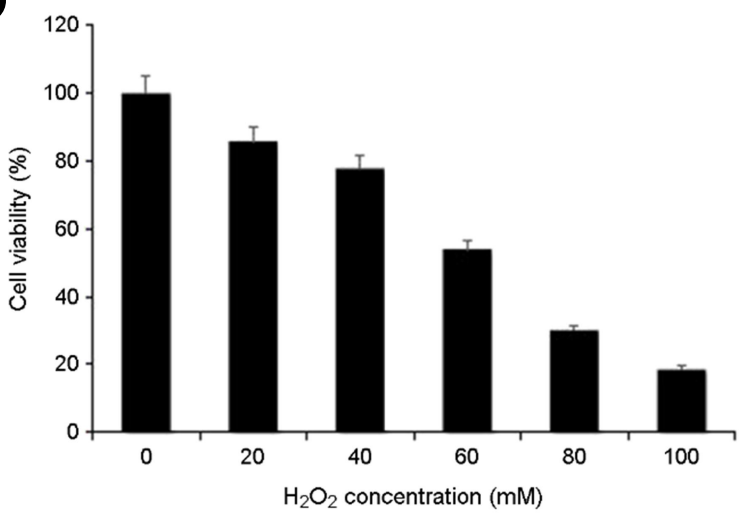

B

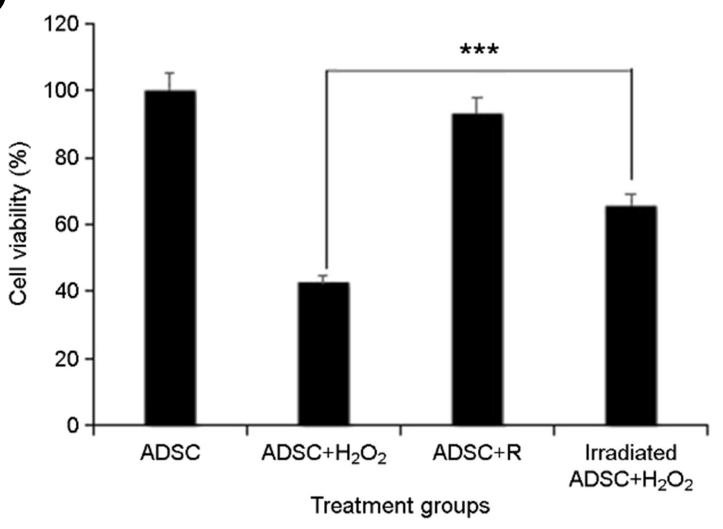

Fig. 3. Anti-oxidant effect of ANXA1 expressed by low-dose radiation under oxidant stress condition. (A) Optimal concentration of $\mathrm{H}_{2} \mathrm{O}_{2}$ to mimic oxidative environment in ADSC. Stem cells were treated with $\mathrm{H}_{2} \mathrm{O}_{2}$ at various concentrations for $4 \mathrm{~h}$, and then the $\mathrm{IC}_{50}$ of $60 \mathrm{mM}$ concentration was decided. (B) Anti-oxidant effect of ANXA1 expressed by low-dose radiation under oxidative stress

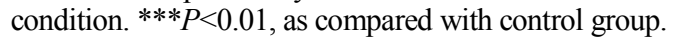

$\mathrm{H}_{2} \mathrm{O}_{2}$-treated group (Fig. 3B). These results indicate that radiation-induced ANXA1 induces antioxidant properties in ADSCs.

\section{Improved antioxidant properties of ANXA1-expressing ADSCs}

As shown in the results in Fig. 3, we confirmed that radiation-induced ANXA1 is resistant to oxidative cellular damage. Therefore, we examined whether artificially expressed ANXA1 also exhibits antioxidant properties. Thus, an ANXA1-expressing vector was transfected into ADSCs. Two days after the transfection, the cells were treated with $\mathrm{H}_{2} \mathrm{O}_{2}$ (Fig. 4A). As shown in Fig. 3B, the viability of cells treated with $\mathrm{H}_{2} \mathrm{O}_{2}$ or radiation alone was similar to the results 


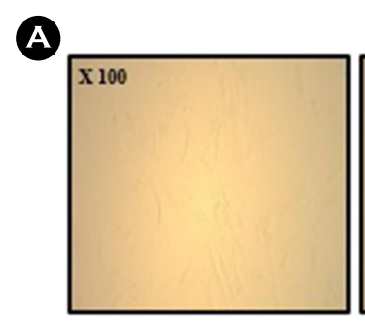

ADSC

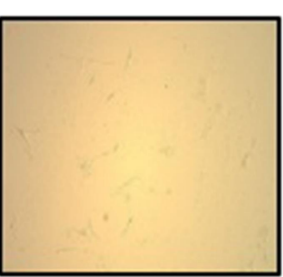

$\mathrm{ADSC}+\mathrm{H}_{2} \mathrm{O}_{2}$

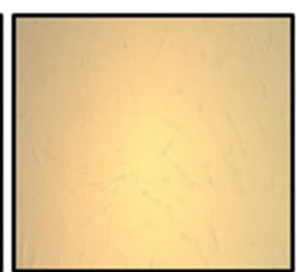

ANXA1 expressing ADSC

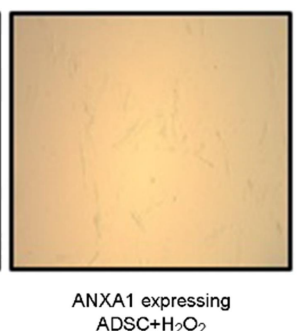

C
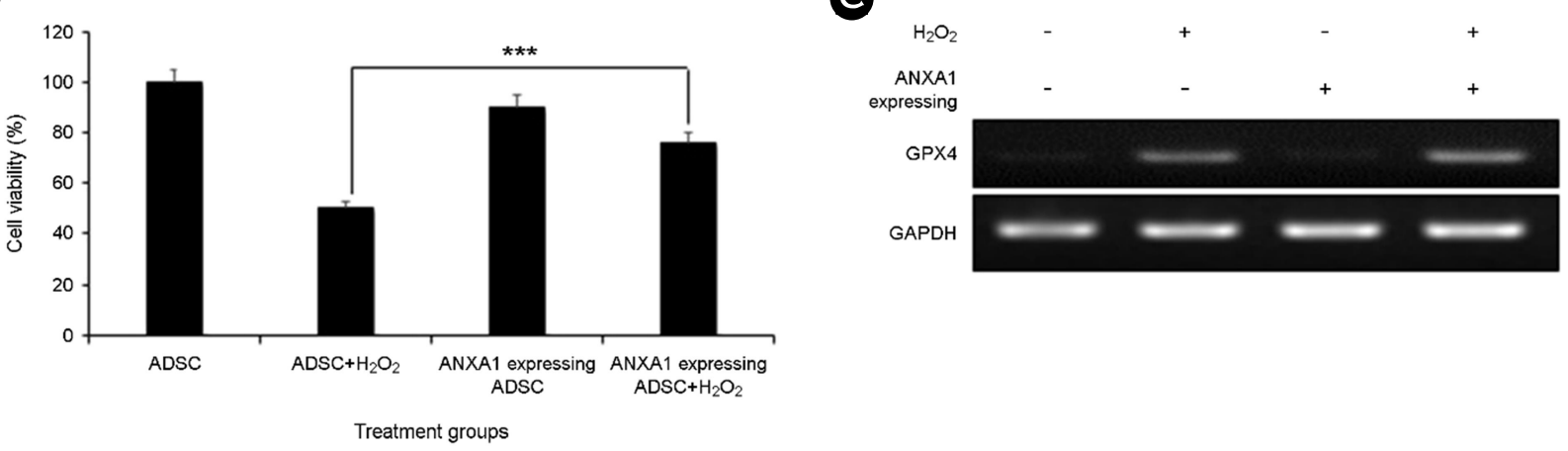

D

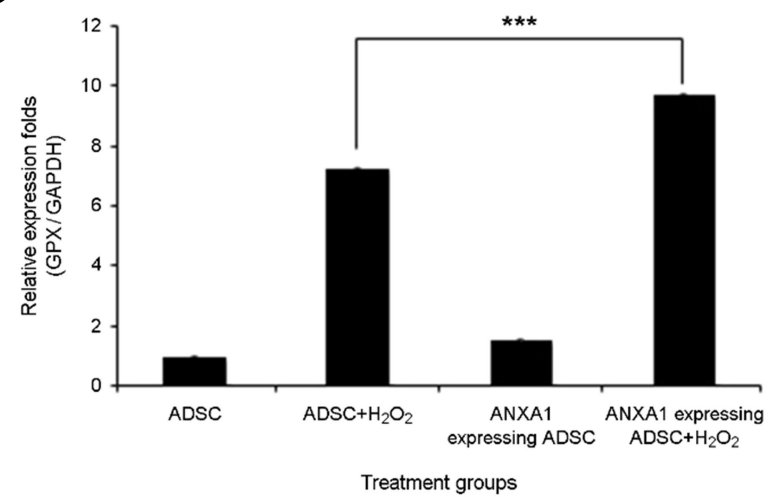

Fig. 4. Enhanced resistant ability against oxidative cellular damage of ANXA1 overexpressing ADSC. (A-B) The antioxidant effect of ANXA1 overexpressed in ADSC. ANXA1 overexpressing vector was transfected to ADSCs. At 2 days post-transfection, ADSCs were exposure in the presence of $\mathrm{H}_{2} \mathrm{O}_{2}$ for $4 \mathrm{~h}$, and then cell viability was measured by MTT assay. (C) Increased reduction ability via the high expression of antioxidant enzyme in ANXA1 expressing ADSCs. After transfection of ANXA1 into ADSC, GPX4 expression was evaluated at the mRNA level. (D) Quantifications of ANXA1 of conventional PCR intensities normalized to their respective controls (defined as 1.0). ${ }^{* * *} P<0.01$, as compared with control group. shown in Fig. 3B. However, ADSCs transfected with the ANXA1 expression vector exhibited a higher survival rate compared with un-transfected ADSCs treated with $\mathrm{H}_{2} \mathrm{O}_{2}$. This indicates that artificially expressed ANXA1 is also resistant to oxidative stress, leading to increased stem cell survival. Next, to confirm the mechanism behind the antioxidant effect shown in Fig. 4B, the expression of the antioxidant enzyme glutathione peroxidase 4 (GPX4) was evaluated. GPX4 catalyzes the reduction of $\mathrm{H}_{2} \mathrm{O}_{2}$ to protect cells against peroxidation and oxidative stress. As shown in Fig. 4C-D, GPX4 expression exhibited a trend similar to that of cell survival (Fig. 4B). GPX4 expression was lower in $\mathrm{H}_{2} \mathrm{O}_{2}$-treated cells than in controls. In contrast, ANXA1-overexpressing ADSCs exhibited the highest GPX4 expression level in all groups, causing an enhanced antioxidant effect.

Taken together, the results of this study demonstrate that ANXA1 acts as a potent antioxidant under oxidative stress conditions.

\section{DISCUSSION}

Radiation therapy, one of the avenues for treatment of malignant tumors, prolongs patient survival (Baskar et al., 2012; Perez and Mutic, 2013). However, damage to normal 
tissue surrounding tumors remains an important clinical concern (Hur and Yoon, 2017). Ionizing radiation, used to treat cancer, affects cancer cells as well as normal cells, including resident stem cells adjacent to the tumor (Narayanan et al., 1997). If normal cells near the tumor are damaged by radiation exposure, serious complications can occur (Bentzen, 2006). It is important to address or prevent radiation-induced cytotoxicity to normal tissue, as the severity of radiationinduced toxicity can be worse than the initial lesions treated. Recent advances in stem cell biology have demonstrated that exposure of stem cells to the genotoxic stress of lowdose ionizing radiation can improve the therapeutic efficacy by repairing damaged cells. However, high-dose radiation can induce death of resident stem cells before they exhibit a positive effect on therapeutic efficacy, and tissue regeneration and recovery (Ciccia and Elledge, 2010; Le et al., 2017).

In this study, we focused on the radiation-induced expression of the ANXA1 gene in ADSCs. This gene is involved in various biological processes, such as inflammation, apoptosis, and calcium transfer signal pathways (Huang et al., 2016). However, its function in stem cells is not well known. Thus, we examined the radiation-induced expression of ANXA1 because no studies have reported its biological function in stem cells.

First, we assessed whether ANXA1 expression is induced by low-dose radiation. We found that ANXA1 levels were upregulated $6 \mathrm{~h}$ after ADSCs were irradiated with $1 \mathrm{mGy}$ radiation (Fig. 2). This result indicates that low-dose radiation induced the expression of ANXA1 in ADSC.

Radiation is used primarily in clinical as a method to treat cancer. However, the treatment also affects normal cells, including resident stem cells, around the irradiated site. Thus, it is important to investigate its effect on normal cells to reduce the severity of the side effects of radiation therapy (Baskar et al., 2012; Peters et al., 2000). Exposure to radiation has been documented to increase stress in affected regions (Benitez et al., 2004). Specifically, oxidative stress induced by radiation has been documented to decrease the viability of the exposed cells. Therefore, we examined whether ANXA1 exhibits anti-oxidative properties in stem cells. The oxidative environment was mimicked by treating ADSCs with $\mathrm{H}_{2} \mathrm{O}_{2}$ (Fig. 3). Our results indicated that radiation- induced expression of ANXA1 improved viability of $\mathrm{H}_{2} \mathrm{O}_{2}$ treated cells compared with that of untreated controls (Fig. 4). This indicates that ANXA1 acts as an antioxidant.

To further clarify the function of ANXA1, we induced overexpression of ANXA1 by transfecting ADSCs with an ANXA1 expression vector. Furthermore, ADSCs overexpressing ANXA1 not only exhibited higher cell survival under oxidative stress conditions, as shown in Fig. 4, but also exhibited higher expression of GPX4.

Taken together, our study suggests that ANXA1 can be used as a potential therapeutic target to enhance the cell viability of ADSCs after radiation treatment.

\section{Abbreviations}

annexin A-1, ANXA1; adipose-derived stem cells, ADSCs; hydrogen peroxide, $\mathrm{H}_{2} \mathrm{O}_{2}$; Low linear energy transfer, LET; reactive oxygen species, ROS; superoxide, $\mathrm{O}_{2}$; hydroxyl radical, $\mathrm{OH}$; glutathione peroxidase 4, GPX4.

\section{ACKNOWLEDGEMENT}

This research was supported by Basic Science Research Program through the National Research Foundation of Korea (NRF) funded by the Ministry of Education (2020R1F1A1053663).

\section{CONFLICT OF INTEREST}

The authors declare that they have no conflict of interest.

\section{REFERENCES}

Baskar R, Lee KA, Yeo R, Yeoh KW. Cancer and radiation therapy: Current advances and future directions. Int J Med Sci. 2012. 9: 193.

Beerman I. Accumulation of DNA damage in the aged hematopoietic stem cell compartment. Semin Hematol. 2017. 54: 12.

Benitez FJ, Acero JL, Real FJ, Roman S. Oxidation of mcpa and 2, 4-d by uv radiation, ozone, and the combinations uv/h2o2 and $\mathrm{o} / \mathrm{h} 2 \mathrm{o} 2$. Journal of Environmental Science and Health, Part B. 2004. 39: 393-409.

Bentzen SM. Preventing or reducing late side effects of radiation therapy: Radiobiology meets molecular pathology. Nature Reviews Cancer. 2006. 6: 702-713.

Buldak R, Gowarzewski M, Buldak L, Skonieczna M, Kukla M, 
Polaniak R, et al. Viability and oxidative response of human colorectal het-116 cancer cells treated with visfatin/enampt in vitro. J Physiol Pharmacol. 2015. 66: 557-566.

Ciccia A, Elledge SJ. The DNA damage response: Making it safe to play with knives. Mol Cell. 2010. 40: 179-204.

Huang L, Liao L, Wan Y, Cheng A, Li M, Chen S, et al. Downregulation of annexin a1 is correlated with radioresistance in nasopharyngeal carcinoma. Oncol Lett. 2016. 12: 5229-5234.

Hur W, Yoon SK. Molecular pathogenesis of radiation-induced cell toxicity in stem cells. Int J Mol Sci. 2017. 18. Epub 2017/12/ 21. doi: 10.3390/ijms18122749. PubMed PMID: 29258244; PubMed Central PMCID: PMCPMC5751348.

Koh EY, You JE, Jung SH, Kim PH. Biological function of carcinoembryonic antigen-related cell adhesion molecule 6 for the enhancement of adipose-derived stem cell survival against oxidative stress. Korean Journal of Clinical Laboratory Science. 2019. 51: 475-483.

Le M, Fernandez-Palomo C, McNeill FE, Seymour CB, Rainbow AJ, Mothersill CE. Exosomes are released by bystander cells exposed to radiation-induced biophoton signals: Reconciling the mechanisms mediating the bystander effect. PLoS One. 2017. 12: $\mathrm{e} 0173685$.

Liauw SL, Connell PP, Weichselbaum RR. New paradigms and future challenges in radiation oncology: An update of biological targets and technology. Sci Transl Med. 2013. 5: 173 sr172-173sr172.

Lim LH, Pervaiz S. Annexin 1: The new face of an old molecule. The FASEB Journal. 2007. 21: 968-975.
Mohrin M, Bourke E, Alexander D, Warr MR, Barry-Holson K, Le Beau MM, et al. Hematopoietic stem cell quiescence promotes error-prone DNA repair and mutagenesis. Cell Stem Cell. 2010. 7: 174-185.

Narayanan P, Goodwin EH, Lehnert B. A particles initiate biological production of superoxide anions and hydrogen peroxide in human cells. Cancer Res. 1997. 57: 3963-3971.

Onmaz A, Van Den Hoven R, Gunes V, Cinar M, Kucuk O. Oxidative stress in horses after a 12-hours transport period. Rev Med Vet. 2011. 162: 213-217.

Perez CA, Mutic S. Advances and future of radiation oncology. Reports of Practical Oncology \& Radiotherapy. 2013. 18: 329 -332 .

Peters III WA, Liu P, Barrett RJ, Stock RJ, Monk BJ, Berek JS, et al. Concurrent chemotherapy and pelvic radiation therapy compared with pelvic radiation therapy alone as adjuvant therapy after radical surgery in high-risk early-stage cancer of the cervix. Obstet Gynecol Surv. 2000. 55: 491-492.

Seifried HE, Anderson DE, Fisher EI, Milner JA. A review of the interaction among dietary antioxidants and reactive oxygen species. J Nutr Biochem. 2007. 18: 567-579.

https://doi.org/10.15616/BSL.2020.26.4.249

Cite this article as: You JE, Lee SW, Kim KS, Kim PH. Antioxidant Effect of Annexin A-1 Induced by Lowdose Ionizing Radiation in Adipose-derived Stem Cells. Biomedical Science Letters. 2020. 26: 249-255. 